\title{
Dominant Wave Frequency and Amplitude Estimation for Adaptive Control of Wave Energy Converters
}

\author{
Hoai-Nam Nguyen, Paolino Tona and Guillaume Sabiron \\ Control, Signal and System Department \\ IFP Energies Nouvelles, Rond-point de l'echangeur de Solaize BP3, \\ 69360 Solaize, France Email: name.surname@ifp.fr
}

\begin{abstract}
Adaptive control is of great interest for wave energy converters (WEC) due to the inherent time-varying nature of sea conditions. Robust and accurate estimation algorithms are required to improve the knowledge of the current sea state on a wave-to-wave basis in order to ensure power harvesting as close as possible to optimal behavior. In this paper, we present a simple but innovative approach for estimating the wave force dominant frequency and wave force dominant amplitude based on the unscented Kalman filter. The main benefits of the proposed method are illustrated by a numerical example concerning a small-scale wave energy converter prototype, with data coming from experimental recordings obtained in a test basin with irregular waves.
\end{abstract}

\section{INTRODUCTION}

Precise knowledge of current sea-state characteristics is crucial in advanced control methods for wave energy converters (WECs). More specifically, having real-time information at disposal about dominant frequency and dominant amplitude of the wave excitation force acting on the device, enables the controller to calculate the control action in an optimal manner.

Les us recall that the ultimate objective in the hydrodynamic control of a WEC is the maximisation, for a broad range of sea states, of the energy captured from the waves, via the force applied by the power-take off (PTO) system (or secondary converter) to the primary converter $\left(f_{P T O}\right.$ in Fig. 1 which provides a simplified representation of a heaving-buoy wave energy converter). The reader can refer to [1] for an extended definition of primary and secondary converters.

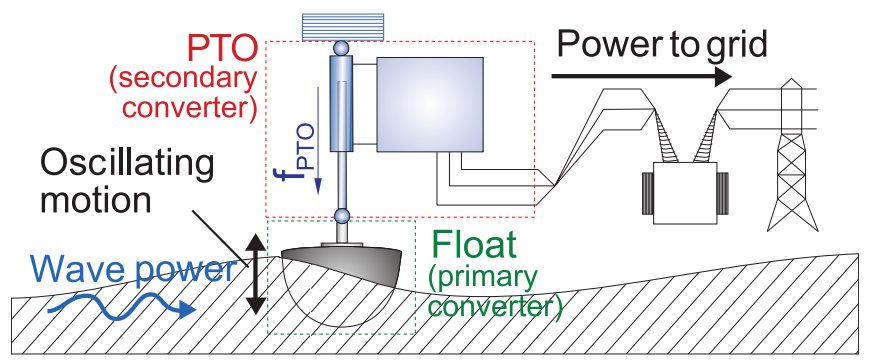

Fig. 1. Schematic diagram of a generic (heaving-buoy) wave energy converter

Latching control, model predictive control (MPC) and PID control are examples of implementable WEC control strategies [2].

For WECs with a flexible PTO, capable of both harvesting and drawing power from the grid (respectively in generator and motor modes), a commonly adopted solution, due to its simplicity, is the so called PI (or BK) controller described in [3]. This control law consists in computing the PTO force via a combined proportional feedback on the position and velocity of the primary converter (the float on Fig. 1$)^{1}$. The damping controller (or P controller), where the PTO force is specified to be proportional to and oppositely directed to the velocity of the primary converter, is even more widely adopted, as it does not require the use of reactive power.

To maximise WEC performance, the $\mathrm{P}$ and I gains of these controllers cannot be kept constant. They have to be changed following the changes of sea state. Adaptive P or PI control has been already discussed in the literature [4], [5], [6], at different level of details, in the form of a gain-scheduling approach. The main idea is to compute optimal gains for a representative set of sea states. The gains are calculated offline, analytically or numerically, using a gridding approach: for each sea state, the ones leading to the best average power are picked. Then the appropriate control action $f_{P T O}$ is found from a look-up table whose input is the current sea state, which can be identified, for instance, in terms of dominant wave frequency and amplitude, or other characteristics of its spectrum. In most literature, only average online estimations of sea states have been proposed, with time windows of several minutes (10 minutes in [5], 2030 minutes in [6]). Such intermittent adaptive control laws are clearly suboptimal in terms of energy recovery, since the control gains are not continuously updated whereas the sea state is continuously time-varying.

By contrast, an efficient method to estimate online the dominant frequency and dominant amplitude of wave excitation force, would allow to adapt the control gains on a wave-towave basis. This would enable the design of a continuously adaptive (PI) control system such as the one depicted in Fig. 2, provided that the wave force excitation can be estimated online.

An estimation approach based on an Extended Kalman Filter (EKF), applied to a nonstationary, harmonic approximation of the wave excitation force, is proposed in [7] as part of the "simple-but-effective" WEC controller. The proposed approach consists in a velocity tracking controller with adaptive parameters computed using online estimates of the dominant frequency and dominant amplitude of the wave

\footnotetext{
${ }^{1}$ This corresponds to a proportional-integral action on the velocity of the primary converter, which is the reason for it being confusingly referred to as PI controller.
} 


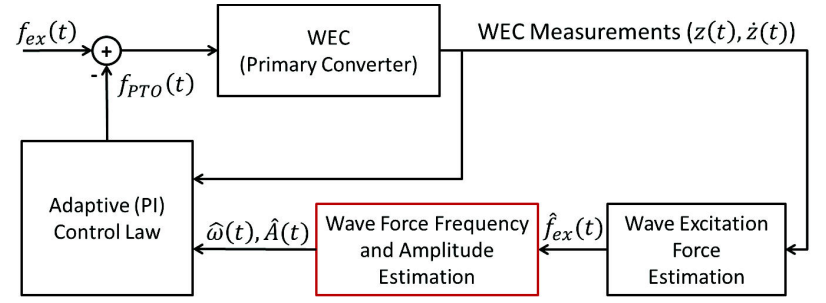

Fig. 2. Adaptive WEC control system with wave force frequency and amplitude estimation

excitation force. The rationale behind the choice of such an estimation approach is that the wave force can be seen as a sum of a number of sinusoids (assuming a panchromatic sea state, [8]), but its dependency on frequency and amplitude is a nonlinear function, preventing the use of standard linear Kalman filter (KF). Moreover, it is quite natural to resort to robust methods to obtain accurate estimates in the presence of noise and/or uncertainties in wave force measurements or estimates.

Nonetheless, it must be recalled that the EKF provides a solution to a nonlinear estimation problem via local linearisations of the underlying model. This entails several drawbacks for this applications. In particular, when the variation of the wave force is large and/or the sampling time intervals are not sufficiently small, linearisation can produce highly unstable filters, potentially leading to divergence phenomena [9], [10] In practice, this jeopardises the ability to accurately track dominant wave frequency and amplitude, when the sea state changes.

To address these limitations, the Unscented Kalman Filter (UKF) is proposed in [9]. This is a powerful nonlinear estimation technique and has been shown to be a superior alternative to the EKF in several applications including parameter estimation for time series modeling [11], neural network training [12], and state estimation for road vehicle navigation [13]. An interesting feature of UKF is that no linearisation of the nonlinear model is required.

Although the UKF has been used in a wide range of estimation problems, to the best of the authors' knowledge there has been no attempt to use it to estimate the dominant frequency and amplitude of the wave excitation force. In this paper, we aim to fill this gap by exploring the potential benefits of the UKF in wave frequency and amplitude estimation. Real data recordings are used to analyse the validity and performance of the proposed UKF algorithm.

This paper is organised as follows. Section 2 covers the problem formulation. Section 3 is dedicated to the unscented Kalman filter design. In Section 4, a state space model, that is used in the UKF, is presented. Results obtained with experimental data are evaluated in Section 5 before drawing the conclusion.

\section{PROBlem FORMULATION}

In this paper we are interested in estimating in real-time the dominant frequency and dominant amplitude for a given wave excitation force acting on the WEC.

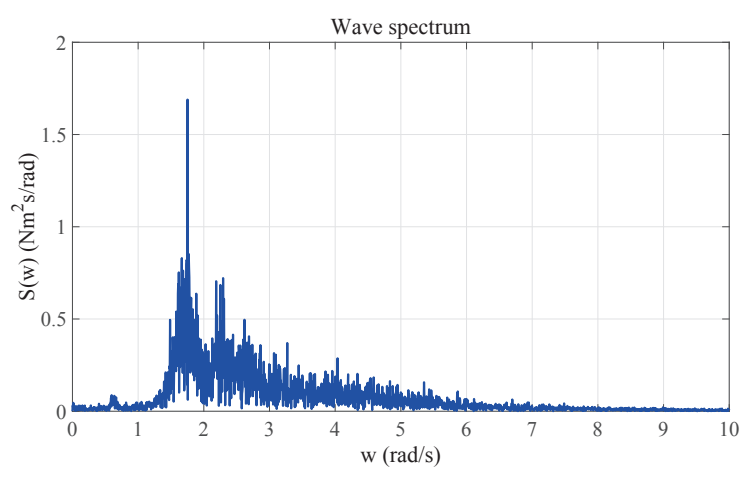

Fig. 3. Typical wave force spectrum for a fully developed sea (experimental data).

The dominant frequency of the wave force is defined as the peak of its spectrum, obtained by using the Fourier transform of the corresponding time signal. For example, in Fig. 3, the dominant wave force frequency is about $1.75 \mathrm{rad} / \mathrm{s}$. The wave force dominant amplitude is defined as the best magnitude, that the wave force can be approximated to by a sinusoid with the dominant frequency and amplitude.

\section{UNSCENTED KALMAN FILTERING}

The main idea behind the unscented Kalman filter is that it is easier to approximate a Gaussian distribution than it is to approximate an arbitrary nonlinear function.

Instead of linearising the nonlinear functions, the UKF uses a deterministic sampling approach to capture the mean and covariance estimates with a minimal set of sample points.

\section{A. Unscented transformation}

The idea of Unscented Transformation (UT) is:

- To choose deterministically $2 n+1$ sample points (also called sigma points) with mean $\bar{x}$ and covariance matrix $P_{x x}$. Here $n$ is the dimension of $x$.

- To find the mean $\bar{y}$ and covariance matrix $P_{y y}$ by propagating the sample points through a nonlinear function.

Define $S$ as Cholesky factorisation of $P_{x x}$, i.e. $P_{x x}=S^{T} S$. In UT, the sample points can be obtained as

$$
\left\{\begin{array}{l}
\chi_{0}=\bar{x} \\
\chi_{i}=\bar{x}+\sqrt{n+\lambda} S_{i}, \quad i=1,2 \ldots, n \\
\chi_{i+n}=\bar{x}-\sqrt{n+\lambda} S_{i}, \quad i=1,2 \ldots, n
\end{array}\right.
$$

where $S_{i}$ is the $i$-th column of the matrix $S$, and $\lambda=\alpha^{2}(n+$ $\kappa)-n$. The scaling parameter $\alpha$ determines the spread of the sample points and $\kappa$ is a secondary scaling parameter.

Once the sample points are computed, we propagate them through the nonlinear function:

$$
\gamma_{i}=f\left(\chi_{i}\right), \quad i=0,1, \ldots, 2 n
$$


The next step is to calculate the mean $\bar{y}$ and the covariance matrix $P_{y y}$ of the propagated points as

$$
\left\{\begin{array}{l}
\bar{y}=\sum_{i=0}^{2 n} W_{i}^{m} \gamma_{i}, \\
P_{y y}=\sum_{i=0}^{2 n} W_{i}^{c}\left(\gamma_{i}-\bar{y}\right)\left(\gamma_{i}-\bar{y}\right)^{T}
\end{array}\right.
$$

where the weights $W_{i}^{m}$ and $W_{i}^{c}$ are

$$
\left\{\begin{array}{l}
W_{0}^{m}=\frac{\lambda}{n+\lambda}, \\
W_{i}^{m}=\frac{1}{2(n+\lambda)}, \\
W_{0}^{c}=\frac{\lambda}{n+\lambda}+1-\alpha^{2}+\beta, \\
W_{i}^{c}=\frac{1}{2(n+\lambda)}
\end{array}\right.
$$

Note that $\beta$ is a parameter used to incorporate any prior knowledge about the distribution of $x$.

\section{B. Unscented Kalman filter}

The UKF is an algorithm which uses an underlying process model to make an estimate of the current state of a system and then corrects the estimate using any available sensor measurements.

Consider the following system

$$
\left\{\begin{array}{l}
x(k)=f(x(k-1))+\nu(k-1), \\
y(k)=g(x(k))+\mu(k)
\end{array}\right.
$$

where $x(k) \in \mathbb{R}^{n}$ is the state vector, $y(k) \in \mathbb{R}^{m}$ is the measurement vector, $\nu(k)$ and $\mu(k)$ are respectively, the process noise and the measurement noise. It is assumed that $\nu(k)$ and $\mu(k)$ are Gaussian noises with zero mean and with covariance matrices $Q$ and $R$, respectively.

For the given state estimate $\hat{x}(k-1)$, and the given covariance matrix estimate $P(k-1)$, there are three steps in the UKF.

1. Sigma point calculation.

Using the state estimate $\hat{x}(k-1)$ and the covariance matrix estimate $P(k-1)$ at time $k-1$, the sigma points are calculated, at time $k$, as

$$
\left\{\begin{array}{l}
\chi_{0}=\hat{x}(k-1) \\
\chi_{i}=\hat{x}(k-1)+\sqrt{n+\lambda} S_{i}, \quad i=1,2 \ldots, n \\
\chi_{i+n}=\hat{x}(k-1)-\sqrt{n+\lambda} S_{i}, \quad i=1,2 \ldots, n
\end{array}\right.
$$

where $S$ is the Cholesky factorisation of $P(k-1)$.

2. State prediction.

The predicted state mean vector $\hat{x}(k \mid k-1)$, and the predicted covariance matrix $P(k \mid k-1)$ are computed as

$$
\left\{\begin{aligned}
\hat{x}(k \mid k-1)= & \sum_{i=0}^{2 n} W_{i}^{m} \gamma_{i} \\
P(k \mid k-1)= & \sum_{i=0}^{2 n} W_{i}^{c}\left(\gamma_{i}-\hat{x}(k \mid k-1)\right)\left(\gamma_{i}-\hat{x}(k \mid k-1)\right)^{T} \\
& +Q
\end{aligned}\right.
$$

where

$$
\gamma_{i}=f\left(\chi_{i}\right), \quad i=0,1, \ldots, 2 n
$$

3. State correction.
The sigma points related to the predicted state mean vector and the covariance matrix are calculated as

$$
\left\{\begin{array}{l}
\psi_{0}=\hat{x}(k \mid k-1) \\
\psi_{i}=\hat{x}(k \mid k-1)+\sqrt{n+\lambda} T_{i}, \quad i=1,2 \ldots, n \\
\psi_{i+n}=\hat{x}(k \mid k-1)-\sqrt{n+\lambda} T_{i}, \quad i=1,2 \ldots, n
\end{array}\right.
$$

where $T$ is the Cholesky factorisation of $P(k \mid k-1)$. The sigma points are then propagated through the output equation:

$$
\zeta_{i}=g\left(\psi_{i}\right)
$$

The following step is to calculate the output mean vector, the output covariance matrix and the cross covariance matrix as

$$
\left\{\begin{array}{l}
\hat{y}(k \mid k-1)=\sum_{i=0}^{2 n} W_{i}^{m} \zeta_{i}, \\
P_{y y}=\sum_{i=0}^{2 n} W_{i}^{c}\left(\zeta_{i}-\hat{y}(k \mid k-1)\right)\left(\zeta_{i}-\hat{y}(k \mid k-1)\right)^{T}+R \\
P_{x y}=\sum_{i=0}^{2 n} W_{i}^{c}\left(\gamma_{i}-\hat{x}(k \mid k-1)\right)\left(\zeta_{i}-\hat{y}(k \mid k-1)\right)^{T}
\end{array}\right.
$$

The Kalman filter gain, the state estimate and the covariance matrix at time $k$ are given by

$$
\left\{\begin{array}{l}
K(k)=P_{x y} P_{y y}^{-1} \\
\hat{x}(k)=\hat{x}(k \mid k-1)+K(k)(y(k)-\hat{y}(k \mid k-1)) \\
P(k)=P(k \mid k-1)-K(k) P_{y y} K(k)^{T}
\end{array}\right.
$$

\section{UKF parameters}

The UKF has five parameters: $Q, R, \alpha, \beta$ and $\kappa$.

The matrices $Q$ and $R$ represent the process noise covariance and the measurement noise covariance. $R$ is determined empirically and accounts for the uncertainty in the output measurements. Choosing the process noise covariance matrix is not straightforward. In general, there is no systematic way to calculate $Q$.

The scale parameter $\alpha$ determines the spread of sigma points and is set to be $10^{-3}$ in this work. $\beta$ is used to incorporate prior knowledge of the distribution of $x$; for a Gaussian distribution, $\beta=2$ is optimal. In this work, $\kappa=0$ is used.

\section{StATE SPACE MODEL}

It is assumed that the wave excitation force can be modeled as a time varying sinusoidal signal, i.e.,

$$
f_{\text {ex }}(t)=A(t) \sin (\omega(t) t+\phi(t))+\mu(t)
$$

where $f_{e x}(t)$ is the wave force at time $t, A(t)$ is the timevarying dominant magnitude, $\omega(t)$ is the time-varying dominant frequency, $\phi(t)$ is the time-varying phase angle, and $\mu(t)$ is a zero mean random noise. In this model the other harmonics are considered as a random noise.

A state space model is needed for the UKF. Assume that $f_{e x}(t)$ is measured and/or estimated at the times $k T_{s}$, where $k=0,1,2, \ldots$, and $T_{s}$ is the sampling time. Define the following three states :

$$
\left\{\begin{array}{l}
x_{1}(k)=A \sin \left(k T_{s} \omega+\phi\right) \\
x_{2}(k)=A \cos \left(k T_{s} \omega+\phi\right) \\
x_{3}(k)=\omega
\end{array}\right.
$$


Here we reformulate the state equations in a recursive way to cope with UKF requirements. In order to do that, $A, \omega$ and $\varphi$ are considered constant at first. Using the following trigonometric relations

$$
\left\{\begin{array}{l}
\sin (\alpha+\beta)=\sin (\alpha) \cos (\beta)+\cos (\alpha) \sin (\beta) \\
\cos (\alpha+\beta)=\cos (\alpha) \cos (\beta)-\sin (\alpha) \sin (\beta)
\end{array}\right.
$$

one has

$$
\begin{gathered}
x_{1}(k)=A \sin \left((k-1) T_{s} \omega+T_{s} \omega+\phi\right) \\
=A \sin \left((k-1) T_{s} \omega+\phi\right) \cos \left(T_{s} \omega\right)+ \\
\quad+A \cos \left((k-1) T_{s} \omega+\phi\right) \sin \left(T_{s} \omega\right) \\
=\cos \left(T_{s} x_{3}(k-1)\right) x_{1}(k-1)+ \\
\quad+\sin \left(T_{s} x_{3}(k-1)\right) x_{2}(k-1)
\end{gathered}
$$

and

$$
\begin{aligned}
x_{2}(k)= & A \cos \left((k-1) T_{s} \omega+T_{s} \omega+\phi\right) \\
= & A \cos \left((k-1) T_{s} \omega+\phi\right) \cos \left(T_{s} \omega\right)- \\
& -A \sin \left((k-1) T_{s} \omega+\phi\right) \sin \left(T_{s} \omega\right) \\
=- & \sin \left(T_{s} x_{3}(k-1)\right) x_{1}(k-1)+ \\
& \quad+\cos \left(T_{s} x_{3}(k-1)\right) x_{2}(k-1)
\end{aligned}
$$

Denote

$$
x(k)=\left[\begin{array}{lll}
x_{1}(k) & x_{2}(k) & x_{3}(k)
\end{array}\right]^{T}
$$

Denote also

$$
A_{x}=\left[\begin{array}{rrr}
\cos \left(T_{s} x_{3}(k-1)\right) & \sin \left(T_{s} x_{3}(k-1)\right) & 0 \\
-\sin \left(T_{s} x_{3}(k-1)\right) & \cos \left(T_{s} x_{3}(k-1)\right) & 0 \\
0 & 0 & 1
\end{array}\right]
$$

Combining (16), (17), and assuming $\omega(k)$ changes slowly with the time, the following state space model is obtained

$$
x(k)=A_{x} x(k+1)+\nu(k)
$$

where

$$
\nu(k)=\left[\begin{array}{lll}
\nu_{1}(k) & \nu_{2}(k) & \nu_{3}(k)
\end{array}\right]^{T}
$$

Process noises $\nu(k-1)$ are included in the state equation (19) to take into account the time varying nature of $A(k), \omega(k)$ and $\varphi(k)$, although (16), (17) are exact. This is explained by the fact that (16), (17) do not take into consideration the timevarying nature of $A(t), \omega(t)$, and $\phi(t)$. Hence $\nu_{1}(k-1), \nu_{2}(k-$ $1)$ are used to model these unknown uncertainties. Clearly, $\nu_{1}(k-1), \nu_{2}(k-1)$ and $\nu_{3}(k-1)$ are correlated.

For the output equation, denoting $y(k)=f_{\text {ex }}(k)$ and

$$
C=\left[\begin{array}{lll}
1 & 0 & 0
\end{array}\right],
$$

one obtains

$$
y(k)=C x(k)+\mu(k)
$$

where $\mu(k)$ is used to model the measurement error in the output equation and to take into account the fact that $f_{e x}(k)$ contains other harmonics.

Combining (19), (20), one obtains,

$$
\left\{\begin{array}{l}
x(k)=A_{x} x(k-1)+\nu(k-1) \\
y(k)=C x(k)+\mu(k)
\end{array}\right.
$$

The UKF presented in section III.B is then applied to the nonlinear system defined in (21) to provide estimates of $x_{1}(k)$, $x_{2}(k)$ and $x_{3}(k)$. From the estimates, one can then derive dominant wave force frequency estimation and dominant wave force amplitude estimation:

$$
\hat{\omega}(k)=\hat{x}_{3}(k)
$$

Note that the magnitude $A(k)$ is not a state of the system (21). However, once $\hat{x}(k)$ is available, it can be easily calculated as

$$
\hat{A}(k)=\sqrt{\hat{x}_{1}(k)^{2}+\hat{x}_{2}(k)^{2}}
$$

\section{EXPERIMENTAL STUDY}

\section{A. Experimental setup}

The experimental work was carried out in a wave basin of Aalborg University (AAU), Denmark, see Fig. 4. It consists of four parts: the wave generation (a), the wave propagation zone (b), the point absorber laboratory model (c), and the wave termination (d). Clearly, the wave generator and the point absorber are the main interest in the context of this work.

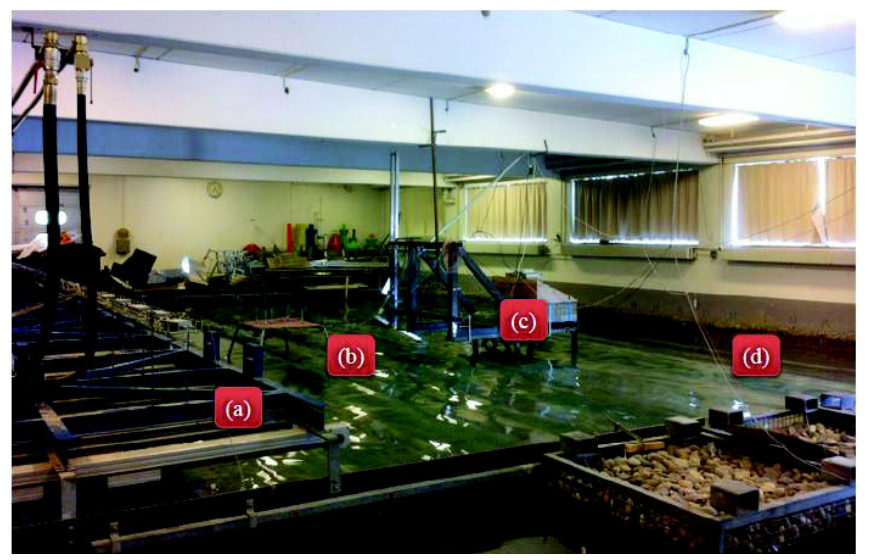

Fig. 4. Experimental setup at AAU. The waves are generated by the wave generation system (a) and the wave elevation is measured by a series of wave gauges (b). The waves then reach the WEC laboratory model, where the wave energy absorption takes place (c). Finally, they reach the artificial beach where the termination takes place by a scattering process (d).

The basin has a length of $15 \mathrm{~m}$, a width of $8 \mathrm{~m}$ and a maximum water depth of $0.7 \mathrm{~m}$. The wave paddles are driven by a total of 15 hydraulic pistons moving in the horizontal direction. The waves were generated by the wave maker based on a Pierson-Moskowitz spectrum.

\section{B. Wave excitation force estimation}

Note that the wave excitation force $f_{e x}(t)$ is considered to be a known input for the UKF. However, this quantity cannot be directly measured during normal operation. Hence $f_{e x}(t)$ has to be estimated via the measurements of other quantities.

In [14], using a combination of a bank of independent harmonic oscillators and a Luenberger observer, an estimation algorithm for wave excitation force is proposed. The strategy is tested on a real WEC system. However the reported experimental results show a relatively high phase lag in the estimated signal compared to the measured signal. 
In [15], by considering the wave excitation force as a timevarying sinusoid, an EKF approach is presented ${ }^{2}$. However, no experimental results are reported. In addition, the approach can clearly be effective only for very narrow-banded wave forces.

By combining several pressure sensors at discrete points on the buoy surface with the buoy heave position, and with an extended Kalman filter, another approach is proposed in [16]. However, the computational complexity may be high. In addition, several extra pressure sensors are required.

In [17], the authors have reported the successful implementation of a new estimation method developed to overcome the aforementioned drawbacks.

Its most important features, in the context studied here, are:

1) only standard WEC measurements (position, velocity, PTO force) are used by the algorithm.

2) the experimental results show that estimated wave torque values do not have any significant lag compared to "true" values, which is not the case for the approach in [14].

3 ) in contrast to [14], no (implicit) unrealistic assumption about the time-invariant nature of the sea state is made, hence any operating condition can be efficiently dealt with.

The approach is based on a linear Kalman filter and a random walk model for the variation of the wave excitation force. Since wave force estimation is not the main subject of the paper, it will not be discussed further. For more details, the interested reader is referred to [18].

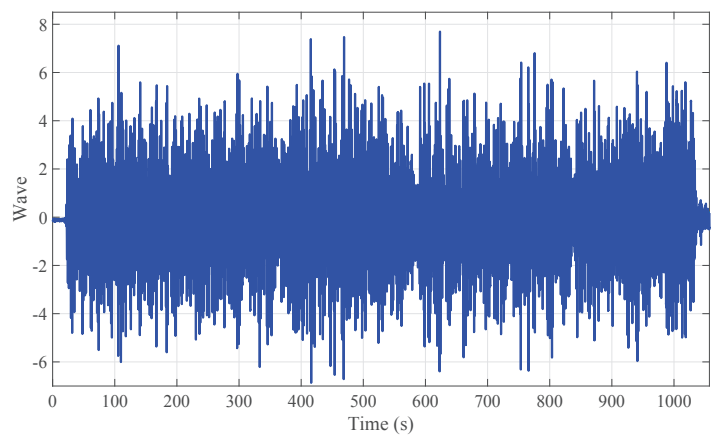

Fig. 5. Irregular wave.

\section{Validation on experimental data}

For the present analysis, an irregular wave is considered, see Fig. 5. The wave is unidirectional, two dimensional and long-crested. The data set is measured at a sampling frequency of $1 \mathrm{kHz}$. To reduce the computational complexity, data downsampling with a factor of 100 is applied. The spectrum of the data set is presented in Fig. 6.

In Fig. 6, it can be observed that the dominant frequency is around $5 \mathrm{rad} / \mathrm{s}$.

${ }^{2}$ Note that the EKF approach mentioned here is applied to the estimation of wave excitation force from WEC measurement, and bears no relation to the UKF approach for the estimation of wave force dominant frequency and amplitude described in this paper, or the EKF mentionned in [7]

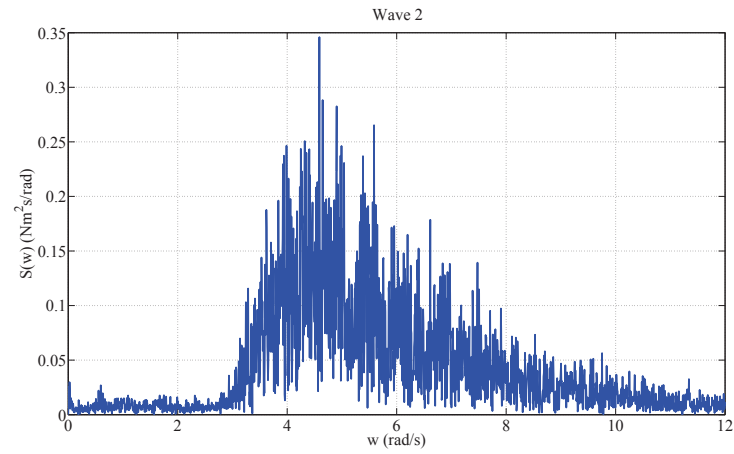

Fig. 6. Spectrum of sample data set.

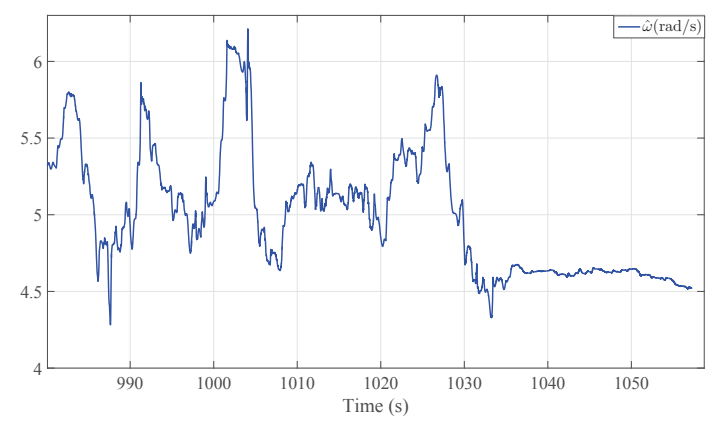

Fig. 7. Dominant frequency estimation.

This fact is confirmed by the dominant frequency estimate shown in Fig. 7.

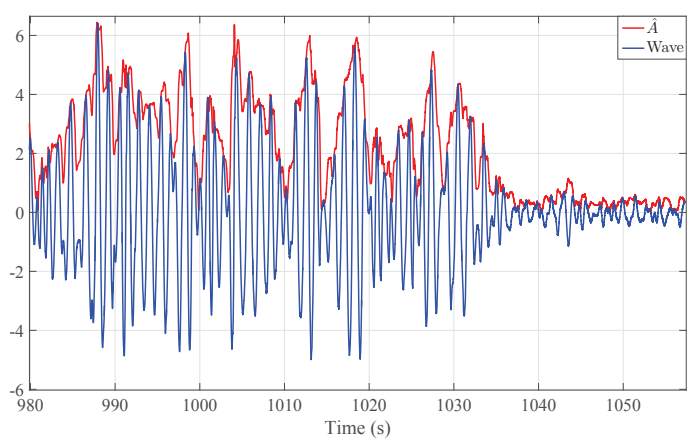

Fig. 8. Dominant magnitude estimation.

Finally, Fig. 8 shows the dominant magnitude estimate. An interesting feature of the UKF is that it is able to estimate the dominant frequency and dominant magnitude even in presence of significant variations of the wave force. This feature can hardly be guaranteed with the EKF.

\section{CONCLUSION}

A simple solution based on the unscented Kalman filter to the problem of estimating the wave force dominant frequency and dominant amplitude has been proposed in this paper. 
Various experimental results have been presented to assess the performance of the UKF. It has been shown that the UKF estimates with high accuracy and no significant lag the dominant frequency and dominant amplitude in spite of significant variations of the wave excitation force. This innovative algorithm thus paves the way to fully functional adaptive control for wave energy converters of the heavingbuoy type (and possibly of other types). It is interesting to note that the proposed algorithm is robust with respect to WEC architecture since the dynamic behaviour of the system does not interfere with the estimation approach and can thus be applied to any kind of WEC system. Future work will focus on estimating residual bias on both wave force dominant amplitude and frequency.

\section{ACKNOWLEDGMENTS}

The authors wish to thank Morten Kramer, Associate Professor at Aalborg University, for providing data on the smallscale protototype and the experimental set-up, and support during the tests.

\section{REFERENCES}

[1] U. Korde and J. Ringwood, Hydrodynamic Control of Wave Energy Devices. Cambridge University Press, 2016.

[2] J. Ringwood, "Control Optimisation and Parametric Design," in Numer ical Modelling of Wave Energy Converters, M. Folley, Ed. Academic Press, 2016, pp. $229-251$

[3] E. V. Sanchez, R. H. Hansen, and M. M. Kramer, "Control performance assessment and design of optimal control to harvest ocean energy," Oceanic Engineering, IEEE Journal of, vol. 40, no. 1, pp. 15-26, Jan 2015.

[4] T. Whittaker, D. Collier, M. Folley, M. Osterried, A. Henry, and M. Crowley, "The development of oystera shallow water surging wave energy converter," in Proceedings of the 7th European Wave and Tidal Energy Conference, Porto, Portugal, 2007, pp. 11-14.

[5] R. H. Hansen and M. M. Kramer, "Modelling and control of the wavestar prototype," in Proceedings of 2011 European Wave and Tidal Energy Conference, 2011.
[6] J. Hals, J. Falnes, and T. Moan, "A comparison of selected strategies for adaptive control of wave energy converters," Journal of Offshore Mechanics and Arctic Engineering, vol. 133, no. 3, p. 031101, 2011.

[7] F. Fusco and J. V. Ringwood, "A simple and effective real-time controller for wave energy converters," IEEE Transactions on sustainable energy, vol. 4, no. 1, pp. 21-30, 2013.

[8] J. Falnes, Ocean waves and oscillating systems: linear interactions including wave-energy extraction. Cambridge university press, 2002.

9] S. J. Julier, J. K. Uhlmann, and H. F. Durrant-Whyte, "A new approach for filtering nonlinear systems," in American Control Conference, Proceedings of the 1995, vol. 3. IEEE, 1995, pp. 1628-1632.

[10] S. J. Julier and J. K. Uhlmann, "New extension of the Kalman filter to nonlinear systems," in AeroSense'97. International Society for Optics and Photonics, 1997, pp. 182-193.

[11] E. A. Wan and R. Van Der Merwe, "The unscented kalman filter for nonlinear estimation," in Adaptive Systems for Signal Processing, Communications, and Control Symposium 2000. AS-SPCC. The IEEE 2000. Ieee, 2000, pp. 153-158.

[12] R. Van Der Merwe and E. A. Wan, "Efficient derivative-free Kalman filters for online learning." in ESANN. Citeseer, 2001, pp. 205-210.

[13] S. J. Julier and H. F. Durrant-Whyte, "Navigation and parameter estimation of high speed road vehicles," in Robotics and automation conference, 1995, pp. 101-105.

[14] P. Kracht, S. Perez-Becker, J.-B. Richard, and B. Fischer, "Performance improvement of a point absorber wave energy converter by application of an observer-based control: Results from wave tank testing," IEEE Transactions on Industry Applications, vol. 51, no. 4, pp. 3426-3434, 2015.

[15] B. Ling and B. Batten, "Real time estimation and prediction of wave excitation forces on a heaving body," in SME. International Conference on Offshore Mechanics and Arctic Engineering, Volume 9: Ocean Renewable Energy, 2015.

[16] O. Abdelkhalik, S. Zou, R. Robinett, G. Bacelli, and D. Wilson, "Estimation of excitation forces for wave energy converters control using pressure measurements," International Journal of Control, pp. 1-13, 2016.

[17] H.-N. Nguyen, G. Sabiron, P. Tona, M. Kramer, and E. Vidal-Sanchez, "Experimental validation of a nonlinear MPC strategy for a wave energy converter prototype," in ASME International Conference on Offshore Mechanics and Arctic Engineering, 2016.

[18] H.-N. Nguyen and P. Tona, "Wave excitation force estimation for wave energy converters of the point absorber type," IEEE Transactions on Control System Technology, 2017, (submitted for publication). 